\title{
Optimization of output coupling ratio on the performance of a ring-cavity Brillouin- erbium fiber laser
}

\begin{abstract}
The operation of a single-wavelength Brillouin-erbium fiber laser (BEFL) system with a Brillouin pump preamplified technique for different output coupling ratios in a ring cavity is experimentally demonstrated. The characteristics of Brillouin Stokes power and tunability were investigated in this research. The efficiency of the BEFL operation was obtained at an optimum output coupling ratio of $95 \%$. By fixing the Brillouin pump wavelength at $1550 \mathrm{~nm}$ while its power was set at $1.6 \mathrm{~mW}$ and the 1480 pump power was set to its maximum value of $135 \mathrm{~mW}$, the Brillioun Stokes power was found to be $28.7 \mathrm{~mW}$. The Stokes signal can be tuned within a range of $60 \mathrm{~nm}$ from 1520 to $1580 \mathrm{~nm}$ without appearances of the self-lasing cavity modes in the laser system.
\end{abstract}

Keyword: Brillouin scattering; Erbium; Fiber laser 